Case Report

\title{
The Use of ECMO in Treatment of Post Liver Transplant Septic Shock
}

\author{
Marina Moguilevitch ${ }^{+,}{ }^{*}$, Michael Rufino ${ }^{\dagger}$, Shalom Frager ${ }^{\dagger}$, Tracey Straker $^{\dagger}$ \\ Montefiore Medical Center, 111 East 210 th street, Bronx, NY 10467, USA; E-Mails: \\ mmoguile@montefiore.org; $\quad$ mrufino@montefiore.org; shfrager@montefiore.org;
}

tstraker@montefiore.org

$\dagger$ These authors contributed equally to this work.

* Correspondence: Marina Moguilevitch; E-Mail: mmoguile@montefiore.org

Academic Editor: Yasuhiko Sugawara

Collection: Infections in Liver Transplantation

OBM Transplantation

2020, volume 4, issue 3

doi:10.21926/obm.transplant.2003116
Received: May 28, 2020

Accepted: August 11, 2020

Published: August 16, 2020

\begin{abstract}
Liver transplant is a life-saving therapy for patients with end stage liver disease and liver cancer. Liver re-transplantation cases are usually more challenging and riskier than primary surgery, they require careful consideration and larger recourse utilization. Decision making on who receives transplantation surgery includes individual patient risks and benefits, physician judgment, specific transplant center policies, surgical experience, geographic organ availability, and ethical considerations. Several mathematical models have been developed to predict survival after re-transplantation. All models show that survival is better in younger patients with greater than two months between transplants, and surgery done before severe decompensation of the patient. The most common cause of early postoperative complications and death after these surgeries was infection leading to sepsis, and very often to septic shock. Early recognition and treatment of sepsis will benefit patient survival after re-transplantation, and lessen the economical burden related to prolonged hospital stay. A controversial topic in the treatment of septic shock remains the role and
\end{abstract}

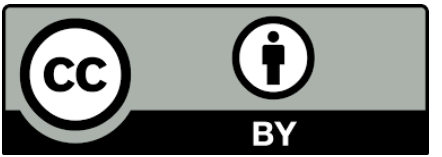

(C) 2020 by the author. This is an open access article distributed under the conditions of the Creative Commons by Attribution License, which permits unrestricted use, distribution, and reproduction in any medium or format, provided the original work is correctly cited. 
utility of extracorporeal membrane oxygenation (ECMO). Identification of suitable candidates for this invasive treatment needs to be determined.

\section{Keywords}

Liver transplant; post-liver transplant complications; septic shock; ECMO

\section{Introduction}

Infection remains a common cause of morbidity and mortality after orthotopic liver transplantation (OLT) [1-3]. Severe infections after liver transplantation can often lead to septic shock with multiorgan failure. Early recognition and introduction of multimodal therapy for septic shock is crucial in the survival of this vulnerable group of patients. There is robust literature describing important steps in addressing septic complications after liver transplantation [4, 5]. The first step involves utilization of broad-spectrum antibiotics and antifungal medications before culture results become available. Interventions should be directed to controlling the source of infection. Hemodynamic and respiratory support to correct hypoxia, hypotension, and impaired tissue oxygenation are paramount.

There are case reports and case series published on utilization of extracorporeal membrane oxygenator (ECMO) before, during, and after liver transplantation [6-8]. Data related to indications, contraindications, and utility of this treatment is scarce. The potential role of ECMO after liver transplantation for patients in septic shock is yet to be carefully examined.

We would like to share our experience with the use of ECMO in the management of a patient who developed septic shock after liver re-transplantation.

\section{Case}

A 22-year-old female with a history of Type 2 autoimmune hepatitis leading to cirrhosis underwent initial orthotopic liver transplantation (OLT) in 2011. Her post-transplant course was complicated by multiple episodes of acute cellular rejection secondary to non-compliance with anti-rejection medications. She was admitted to the hospital prior to surgery for nonhemodynamically significant upper GI bleeding secondary to portal hypertensive gastropathy and esophageal varices. She subsequently developed worsening renal function with creatinine increasing to $5.1 \mathrm{mg} / \mathrm{dL}$ and volume overload. She was transferred to the medical intensive care unit (MICU) on hospital day five for initiation of continuous veno-venous hemodialysis (CVVHD). Her acute on chronic kidney injury was thought to be due to Type 2 hepto-renal syndrome and bile cast nephropathy. During her MICU stay, her liver function progressively deteriorated with total bilirubin increasing to $60 \mathrm{mg} / \mathrm{dL}$ and international normalized ratio (INR) increasing to 1.9. After a multidisciplinary team discussion, considering her young age, and acute worsening of liver and kidney function without feasible recovery, the patient was listed for liver re-transplantation with a MELD score of 42 . All surveillance blood cultures were negative prior to surgery. She was on antimicrobial and antifungal prophylaxis during her entire MICU stay.

The patient underwent re-do of orthotopic liver transplant (OLT) with a brain dead donor graft from a 62 year old Epstein-Barr virus (EBV) Ig positive male. During the procedure, the patient 
received 12 units of blood (PRBC), 6.5 liters cell saver blood, 10 units of fresh frozen plasma (FFP), 2 units of platelets, and 2 units of cryoprecipitate. Standard methylprednisolone and basiliximab induction of immunosuppression was used per institutional protocol. She was started on piperacillin-tazobactam for antimicrobial coverage after the surgery. She returned to operating room for washout and hemostasis on post-op day (POD) 1 . She was hemodynamically stable with normal mental function in the immediate post-operative state, although she noted mild diffuse abdominal pain. The patient continued to require post-operative CVVHD. She was weaned from ventilator support on POD 2 after re-transplantation, but developed low-grade fevers with worsening of abdominal pain and new onset productive cough. On POD3, the patient became progressively hypotensive, tachycardic and tachypnic. She was re-intubated, and initial crystalloid resuscitation started at the rate of $30 \mathrm{ml} / \mathrm{kg}$. To maintain mean arterial pressure in the range $65-70$ $\mathrm{mm} \mathrm{Hg}$, norepinephrine and vasopressin infusions were initiated. Blood cultures were sent, and an urgent flexible sigmoidoscopy performed to rule out $C$. difficile induced megacolon. In addition to piperacillin, she was started on vancomycin and micafungin to ensure broad - spectrum coverage. A bedside echocardiogram was performed, which showed decreased biventricular heart function. Dobutamine was added to improve hemodynamics at the initial rate $10 \mathrm{mcg} / \mathrm{kg} / \mathrm{min}$. Blood cultures returned positive for Klebsiella. Piperacillin and vancomycin were replaced with meropenem, flagyl, and tigecycline. With no significant improvement after six hours of resuscitation, the patient was brought to the operating room for abdominal re-exploration and potential source control.

Fibrinopurulent ascites, patchy ischemic changes of the splenic flexure of the colon, superficial patchy ischemic changes of the spleen, and a congested appearing liver graft with patent vessels was noted on exploration. There was no bile leak or enteric perforation. Upon return from the operating room, the patient remained unstable, and showed no improvement on escalating doses of vasopressor support- norepinephrine drip was at $0.2 \mathrm{mcg} / \mathrm{kg} / \mathrm{min}$, dobutamine $25 \mathrm{mcg} / \mathrm{kg} / \mathrm{min}$, vasopressin 12U/h. Bedside ECHO on ICU arrival demonstrated severe right ventricular hypokinesis, wide open tricuspid regurgitation, and a left ventricular ejection fraction (EF) of $10 \%$. In light of worsening cardiovascular instability secondary to cardiogenic and septic shock, the decision was made to place the patient on venoarterial extracorporeal membrane oxygenator (VA ECMO). ECMO flow was 4.85Lpm, 4300 rpm, sweep 4, FiO2 100\% with a PTT target of 78. Initially, the patient demonstrated hemodynamic improvement, but on POD 4, she became febrile with a temperature of $106 \mathrm{~F}$. On POD 4 she also developed tonic-clonic seizures, and anticonvulsive therapy was initiated. CT scan of the brain demonstrated no significant findings. She had multiple asystolic events treated with epinephrine. Due to progressively worsening acidosis and hyperkalemia, the decision was made to disconnect ECMO support.

\section{Discussion}

Liver transplantation has made significant progress in immunosuppression management, length of ICU stay, length of hospital stay, and postoperative survival. Despite this progress, infections remain the leading cause of critical illness in the perioperative period, increasing morbidity and mortality after liver transplantation $[9,10]$.

Systemic immunosuppression enhances the liver transplantation recipient 's susceptibility to de-novo infections, as well as reactivation of preexisting latent infections. These infections are 
directly correlated with the "net state" of immunosuppression, which is usually highest just after the surgery.

According to the best practice guidelines used by all transplantation programs, patients with active infection should be treated before listing, and those who develop infections while on the transplant list should be held until the infection is eliminated [11].

Usually, infections occurring during first month after OLT are most likely nosocomial, donorderived, or related to perioperative complications. Liver transplant recipients are more prone to developing infections than other transplantation recipients because of the complexity of the surgical procedure, which involves hepatobiliary system manipulation [12]. In the study by Freire et al, the rate of infectious complications was $48.1 \%$. This rate is similar to what was found in previous reports $[13,14]$.

An approach to dealing with infectious complications is to identify specific risk factors for the development of infection, and implement modification of the factors if possible. According to the literature [15-17], there are few major risk factors predisposing transplant candidates to infections: pre-transplant ascites, pre-and post- transplant dialysis, reoperation, wound infection, hepatic artery thrombosis, and Roux-en-Y choledochojejunostomy anastomosis. In addition, The Italian Association for the Study of the Liver disease (Associazione Italiana Studio Del Fegato, AISF) has reported the following risk factors of post-liver transplant infection-model for end stage liver disease (MELD) over 30, level and type of immunosuppression, and primary non-function requiring re-transplantation [18]. The same study did not show any difference of the rate of infection among deceased- and brain dead donor liver recipients. Another study of Varghese et al was able to show a higher rate of infection after deceased-donor transplants [19]. In a sizable Korean study of 222 liver transplant recipients, they identified a $28.8 \%$ blood stream infection (BSI) complications rate [20]. In univariate analysis, the authors were able to show that high MELD score is the strongest predictor of post-operative BSI. In multivariate analysis, they reported biliary complications and longer ICU stay were significant independent factors for post-operative BSI [20].

Bacterial infections were the major cause of infection in studied liver recipients, followed by fungal and viral infections. The most prevalent bacterial species were Enterococcus, followed by $\mathrm{E}$. coli and Klebsiella [21, 22]. Gram-negative bacteria were found to be more prevalent than grampositive in cases of post-liver transplant pneumonia. Fungal infection was noticed more frequently in pre-transplant ICU patients, re-transplantation patients, patients on renal replacement therapy, and patients who received massive transfusions of blood and products (greater than 40 units) [16].

Another important risk factor for post-liver transplantation infection is pre-transplantation infection. If pre-transplantation infection is treated, and post-treatment cultures are negative, up to $70.4 \%$ of these patients have been shown to develop post-transplantation infection with positive cultures [23]. The low detection rate may be explained by empiric use of antibiotics, and difficulty in obtaining an adequate sample. In patients with hepatic failure, concurrent infections can increase the mortality rate by 4-fold [24]. Donor-derived risk factors include infection, prolonged ICU stay before donation, quality of the donor liver, and viral status of the donor [25].

There are several publications $[25,26]$ discussing the role of genetic polymorphism in innate immunity as an important factor that contributes to the infection risk after liver transplantation. This can be a very important mechanism activated in the immunosuppressed patient. This type of immunity includes pattern recognition receptors (PRRs) activation and identification of pathogen- 
associated molecular patterns (PAMPs) on the microorganisms invaded a host. This recognition and activation mechanism kills pathogen directly in lymphocyte -independent manner and activates adaptive immunity. These same studies reinforce the role of toll-like receptors (TLR) and their ability to mount response against gram-negative (TLR 4) and gram-positive (TLR 2) infections in liver transplant patients. Various genetic mutations in the structure of these receptors can impair cytokines secretion and increase risk of infection.

As our knowledge about the mechanisms of infection in immunosuppressed recipients continues to grow, we are challenged by the increasing amount of "marginal" implanted grafts, and the growing need to provide care for sicker patients with multiple co-morbidities. These increased demands spark the possibility of artificial support for respiratory and cardiac function when conventional techniques fail. There are several articles, which analyze the utility of extracorporeal membrane oxygenation (ECMO) before, during, and after liver transplantation [6, 8]. There is universal agreement that ECMO can be offered to patients whose cardiac, pulmonary, or cardio-pulmonary failure is considered to be reversible. The main indication for venovenous (VV) ECMO in these patients is pulmonary failure related to hypoxia in the setting of hepatopulmonary syndrome or porto-pulmonary hypertension. Venoarterial (VA) ECMO was used in cardiac failure related to massive pulmonary embolism (PE) or right heart failure.

There is mounting evidence demonstrating the use of ECMO in septic shock $[27,28]$. Usually, it is reserved for the situations when septic shock is unresponsive to all conventional treatments and targeted antimicrobial therapy. The purpose of using VA ECMO in this condition is to alleviate cardiac dysfunction related to sepsis. In a study by Falk et al, 37 patients were placed on ECMO. The authors observed $90 \%$ survival in septic shock with left ventricular dysfunction, and $64.7 \%$ survival in patients with distributive shock. The results of this study demonstrate clear evidence in favor of ECMO use in sepsis-induced cardiomyopathy. For adults, the use of ECMO in septic shock is not clearly established. While the study by Falk et al shows promising outcomes, Combes [29] demonstrates that indications are still debatable.

In our case, the patient had multiple risk factors for development of post-operative infection and sepsis- in the intensive care unit and while on antibiotic prophylaxis for potential infection prior to her surgery. She was also on renal replacement therapy and scheduled for a liver retransplantation procedure. In addition, she received an extended criteria graft, and had a massive transfusion of blood and blood products. Her young age, healthy heart and lungs were favorable to her outcome. When the patient was presented at the "selection" meeting, there was unanimous agreement among team members that the patient was a suitable candidate with multiple risk factors.

Our experience with the use of post-liver transplantation ECMO in the setting of sepsis-induced cardiomyopathy was unique. The question arises, should ECMO be abandoned for treatment of refractory septic shock after this negative outcome? It is too early to decide. We need to better understand the factors leading to poor outcomes. Gaining more experience in this area, and defining stricter criteria for ECMO utilization may help to provide the best care for this patient population. 


\section{Conclusion}

ECMO is a modality used to give temporary support to failing cardio-pulmonary function in a wide variety of clinical conditions. It has utility during perioperative and intraoperative care for liver transplantation patients with reversible cardio-pulmonary collapse. Our experience with post-liver transplantation and AV ECMO utilized in the setting of sepsis-induced cardiomyopathy may help to define indications, contraindications, and possible limitations in treatment protocol. Further analysis of subsequent cases may allow for prospective interventions.

\section{Acknowledgements}

The authors would like to thank the patient's family for supporting us in sharing this case report.

\section{Authors Contribution}

All authors participated in manuscript creation, review process, and revisions.

\section{Competing Interests}

The authors have declared that no competing interests exist.

\section{References}

1. Blair JE, Kusne S. Bacterial, mycobacterial, and protozoal infections after liver transplantationPart I. Liver Transpl. 2005; 11: 1452-1459.

2. Razonable RR, Paya CV. Liver transplant patients. Inflectional diseases. 3rd ed. London: Mosby Elsevier Science; 2010.

3. Kusne S, Blair JE. Viral and fungal infections after liver transplantation-part II. Liver Transpl. 2006; 12: 2-11.

4. Kalil AC, Opal SM. Sepsis in the severely immunocompromised patient. Curr Infect Dis Rep. 2015; 17: 32.

5. Gea-Banacloche JC, Opal SM, Jorgensen J, Carcillo JA, Sepkowitz KA, Cordonnier C. Sepsis associated with immunosuppressive medications: An evidence-base review. Crit Care Med. 2004; 32: S578-S590.

6. Braun HJ, Pulcarano ME, Weber DJ, Padilla BE, Ascher NL. The utility of ECMO after liver transplantation: Experience at high-volume transplant center and review of the literature. Transplantation. 2019; 103: 1568-1573.

7. Levesque E, Salloum C, Feray C, Azoulay D. The utility of ECMO, not just after but also during liver transplantation. Transplantation. 2019; 103: e319-e320.

8. Auzinger G, Wiliars C, Loveridge R, Vercueil A, Best T, Wendon J. Extracorporate membrane oxygenation before and after adult liver transplantation: Woth the effort? Crit Care. 2014; 18: P203.

9. Fernandez J, Acevedo J, Castro M, Garcia O, Rodríguez de Lope C, Roca D, et al. Prevalence and risk factors of infections by multiresistant bacteria in cirrhosis: A prospective study. Hepatology. 2012; 55: 1551-1561. 
10. Bonnel AR, Bunchorntavakul C, Reddy KR. Immune dysfunction and infections in patients with cirrhosis. Clin Gastroenterol Hepatol. 2011; 9: 727-738.

11. Mah A, Wright A. Infectious considerations in the pre-transplant evaluation of cirrhotic patients awaiting orthotopic liver transplantation. Curr Infect Dis Rep. 2016; 18: 4.

12. Freire MP, Pierrotti LC, Oshiro IC, Bonazzi PR, Oliveira LM, Machado AS, et al. Carbapenemresistant Acinetobacter baumannii acquired before liver transplantation: Impact on recipient outcomes. Liver Transpl. 2016; 22: 615-626.

13. Kim YJ, Kim SI, Wie SH, Kim YR, Hur JA, Choi JY, et al. Infectious complications in living donor liver transplant recipients: A 9-year single-center experience. Transpl Infect Dis. 2008; 10: 316324.

14. Vera A, Contreras F, Guevara F. Incidence and risk factors for infections after liver transplant: Single-center experience at University Hospital Foundation Santa Fe de Bogota, Columbia. Transpl Infect Dis. 2011; 13: 608-615.

15. Arnow PM. Infections following orthotopic liver transplantation. HPB Surg. 1991; 3: 221-233.

16. Chelala L, Kovacs CS, Taege AJ, Hanouneh IA. Common infectious complications of liver transplant. Clev Clin J Med. 2015; 82: 773-784.

17. Lim S, Kim EJ, Lee TB, Choi BH, Park YM, Yang K, et al. Predictors of postoperative infectious complications in liver transplant recipients: Experience of 185 consecutive cases. Korean J Intern Med. 2018; 33: 798-806.

18. Fagiuoli S, Colli A, Bruno R, Craxì A, Gaeta GB, Grossi P, et al. Management of infections preand post-liver transplantation: Report of AISF consensus conference. J Hepatol. 2014; 60: 1075-1089.

19. Varghese J, Gomathy N, Rajashekhar P, Vnugopal K, Olithselvan A, Vivekanandan S, et al. Perioperative bacterial infections in deceased donor and living donor liver transplant recipients. J Clin Exp Hepatol. 2012; 2: 35-41.

20. Kim HK, Park YK, Wang HJ, Kim BW, Shin SY, Lim SK, et al. Epidemiology and clinical features of post-transplant bloodstream infection: An analysis of 222 consecutive liver transplant recipients. Infect Chemother. 2013; 45: 315-324.

21. Kim SI. Bacterial infection after liver transplantation. World J Gastroenterol. 2014; 20: 62116220.

22. Hamandi B, Husain S, Grootendorst P, Papadimitropoulos EA. Clinical and microbiological epidemiology of early and late infectious complications among solid organ transplant recipients requiring hospitalization. Transpl Int. 2016; 29: 1029-1038.

23. Kim YJ, Yoon JH, Kim SI, Choi HJ, Choi JY, Yoon SK, et al. Impact of pretransplant infections on clinical course in liver transplant recipients. Transpl Proc. 2018; 50: 1153-1156.

24. Arvaniti V, D'Amico G, Fede G, Manousou P, Tsochatzis E, Pleguezuelo M, et al. Infections in patients with cirrhosis increase mortality four-fold and should be used in determining prognosis. Gastroenterology. 2010; 139: 1246-1256.

25. Van Hoek B, de Rooij BJ, Verspaget HW. Risk factors for infection after liver transplantation. Best Pract Res Clin Gastroenterol. 2012; 26: 61-72.

26. Razonable RR. Innate immune genetic profile to predict infection risk and outcome after liver transplant. Hepatology. 2010; 52: 814-821.

27. Falk L, Hultman J, Broman LM. Extracorporeal membrane oxygenation for septic shock. Crit Care Med. 2019; 47: 1097-1105. 
28. Myers LC, Lee C, Thompson BT, Cudemus G, Raz Y, Roy N. Outcomes of adult patients with septic shock undergoing extracorporeal membrane oxygenation therapy. Ann Thorac Surg. 2020. Doi: 10.1016/j.athoracsur.2019.12.075.

29. Combes A. Role of VA ECMO in septic shock: Does it work? Quatar Med J. 2017; 2017: 24.

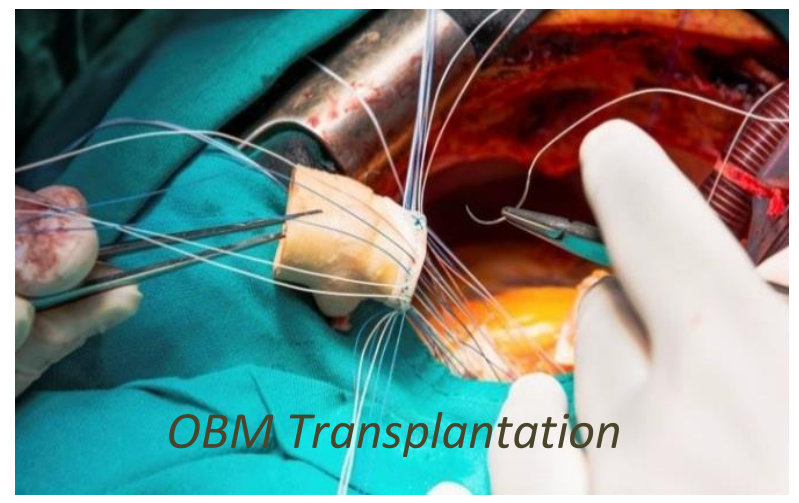

Enjoy OBM Transplantation by:

1. Submitting a manuscript

2. Joining in volunteer reviewer bank

3. Joining Editorial Board

4. Guest editing a special issue

For more details, please visit:

http://www.lidsen.com/journals/transplantation 